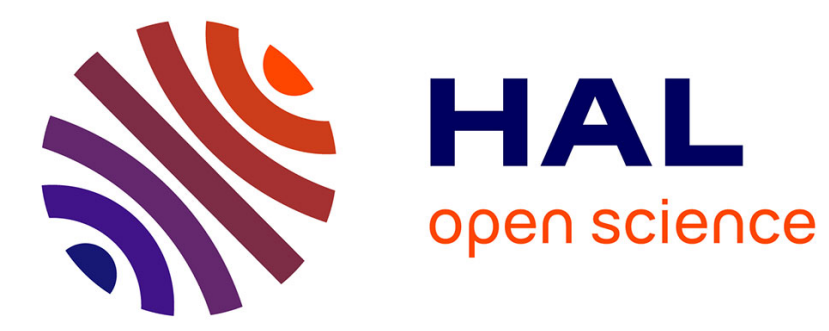

\title{
Onset of cohesion in cement paste
}

B. Jönsson, H. Wennerström, A. Nonat, B. Cabane

\section{To cite this version:}

B. Jönsson, H. Wennerström, A. Nonat, B. Cabane. Onset of cohesion in cement paste. 2004. hal00016073

\section{HAL Id: hal-00016073 \\ https://hal.science/hal-00016073}

Preprint submitted on 17 Dec 2005

HAL is a multi-disciplinary open access archive for the deposit and dissemination of scientific research documents, whether they are published or not. The documents may come from teaching and research institutions in France or abroad, or from public or private research centers.
L'archive ouverte pluridisciplinaire HAL, est destinée au dépôt et à la diffusion de documents scientifiques de niveau recherche, publiés ou non, émanant des établissements d'enseignement et de recherche français ou étrangers, des laboratoires publics ou privés. 


\title{
Onset of cohesion in cement paste
}

\author{
B. Jönsson ${ }^{1}$, H. Wennerström ${ }^{2}$, A. Nonat ${ }^{3}$, B. Cabane ${ }^{4}$
}

1 Theoretical Chemistry, Chemical Center, Lund University, POB 124, S-221 00 Lund, Sweden

2 Physical Chemistry 1, Chemical Center, Lund University, POB 124, S-221 00 Lund, Sweden

3 LRRS, UMR CNRS 5613, Université de Bourgogne, F-21078 Dijon Cedex, France

4 PMMH, ESPCI, 10 rue Vauquelin, F-75231 Paris Cedex 05, France

\begin{abstract}
It is generally agreed that the cohesion of cement paste occurs through the formation of a network of nanoparticles of a calcium-silicate-hydrate ("C-S-H”). However, the mechanism by which these particles develop this cohesion has not been established. Here we propose a dielectric continuum model which includes all ionic interactions within a dispersion of C-S-H particles. It takes into account all co- and counterions explicitly (with pure Coulomb interactions between them and with the surfaces) and makes no further assumptions concerning their hydration or their interactions with the surface sites. At high surface charge densities, the model shows that the surface charge of C-S-H particles is overcompensated by $\mathrm{Ca}^{2+}$ ions, giving a reversal of the apparent particle charge. Also, at high surface charge densities, the model predicts that the correlations of ions located around neighboring particles causes an attraction between the particle surfaces. This attraction has a range of approximately $3 \mathrm{~nm}$ and a magnitude of $1 \mathrm{nN}$, values that are in good agreement with recent AFM experiments. These predictions are stable with respect to small changes in surfacesurface separation, hydrated ion radius, and dielectric constant of the solution. The model also describes the effect of changes in cement composition through the introduction of other ions, either monovalent $(\mathrm{Na})$ or multivalent ( $\mathrm{Al}$ or Fe hydroxide) ions.
\end{abstract}




\section{Introduction}

\section{Cohesion of cement paste}

When Portland cement is mixed with water, anhydrous oxides (mainly $\mathrm{CaO}$ and $\mathrm{SiO}_{2}$ ) react with water to form a solution that contains $\mathrm{Ca}^{2+}, \mathrm{OH}^{-}$and silicate ions $[1,2]$. As the $\mathrm{pH}$ rises above 10 , and the $\mathrm{Ca}^{2+}$ concentration exceeds $1 \mathrm{mM}$, this solution becomes supersaturated with respect to the precipitation of a calcium-silicate-hydrate ("C-S-H"). Nanoparticles of CS-H are formed, with typical dimensions $60 \mathrm{~nm} \cdot 30 \mathrm{~nm} \cdot 5 \mathrm{~nm}$. These particles grow around the cement grains and form a network that fills the spaces between them [1, 2]. Mechanical experiments show that this network is established a few minutes after mixing, and progressively gains strength as hydration proceeds and more C-S-H particles are formed [3]. During hydration, this network maintains similar mechanical properties: it is easily disrupted (critical strain $=0.03 \%$ ) and quickly reformed ( 1 hour after disruption, the elastic modulus has regained the value that it would have reached otherwise). These observations show that the cohesion of cement paste is caused by the same forces throughout the hydration process, the gain in strength resulting from the increase in the amount of C-S-H [3]. Thus, the forces between C-S-H particles are the key to the cohesion of early cement paste.

From a practical point of view, it is extremely important to control the resistance of early cement paste to deformation and flow. For this purpose, we must try to understand the nature of interactions between $\mathrm{C}$-S-H particles. It would also be very useful to be able to predict how these interactions vary with changes in ionic concentrations in the interstitial solution.

\section{$\underline{\text { Ionic conditions in cement paste }}$}

The ionic conditions in cement paste are unusual because of the extremely high concentration of $\mathrm{OH}^{-}$ions ( $\mathrm{pH} 10$ to 13 ). The surface groups of C-S-H particles react with these $\mathrm{OH}^{-}$ions and become negatively charged. The corresponding surface charge density is unusually high, because of the high $\mathrm{OH}^{-}$ion concentration.

In cement paste the charges of $\mathrm{OH}^{-}$ions are balanced by the charges of $\mathrm{Ca}^{2+}$ ions. Because of the precipitation boundary of $\mathrm{Ca}(\mathrm{OH})_{2}$, the bulk concentration of $\mathrm{Ca}^{2+}$ ions is limited $(<20$ $\mathrm{mM}$ at $\mathrm{pH}$ 13) [4]. Since the particles carry a very high negative charge density, there must be a high concentration of $\mathrm{Ca}^{2+}$ ions near the surfaces. Therefore, in the vicinity of $\mathrm{C}-\mathrm{S}-\mathrm{H}$ surfaces, most ions are $\mathrm{Ca}^{2+}$ counterions that compensate the surface charges. 
The interactions of C-S-H particles in this interstitial solution may not be alike usual colloidal interactions, because the surface charge densities are extremely high and divalent counterions are present. There are two main scenarios, which differ according to the behavior of counterions. (i) Either the counterions remain hydrated, and distribute themselves according to the electrical potential in the interstitial solution that separates C-S-H particles. This distribution ("ionic clouds") is usually described by mean-field theories, Poisson-Boltzmann equation and DLVO theory, which predict repulsive forces between surfaces, due to the osmotic pressure of the counterions. (ii) Or the counterions bind chemically to the surface groups, thereby losing some of their hydration. In dilute dispersions, this could lead to reversal of the overall surface charge; in concentrated dispersions it could cause the formation of calcium bridges between surfaces that are quite close to each other. Other possibilities come to mind, such as specific ion binding or surface heterogeneity of the particles, but they are essentially variations of (ii). The problem is that neither of these scenarios can explain the available data.

\section{Experiments}

Recent experiments, performed on C-S-H particles dispersed in an ionic solution that is away form the precipitation boundary (i.e. the particles are not growing) show consistent trends in the surface chemistry of C-S-H according to the concentrations of $\mathrm{OH}^{-}$and $\mathrm{Ca}^{2+}$ ions.

(a) Electrokinetic experiments show that the mobility of the particles changes sign as the pH rises and $\mathrm{Ca}^{2+}$ ions are released in the solution [5]. The particles behave as negatively charged at low concentration of dissolved $\mathrm{Ca}(\mathrm{OH})_{2}(\mathrm{pH}<11.6)$, and positively charged at high concentration of dissolved $\mathrm{Ca}(\mathrm{OH})_{2}(\mathrm{pH}>11.6)$. This reversal of apparent surface charge cannot be explained by mean field models, where the surface charge is only partly compensated by condensed counterions. It would appear to support the hypothesis that $\mathrm{Ca}^{2+}$ ions bind chemically to the surfaces [5]; however, AFM scans of the C-S-H surface do not show any evidence of bound $\mathrm{Ca}^{2+}$ ions.

(b) AFM measurements show that the interparticle force changes sign as the pH rises [6]. In solutions of $\mathrm{pH}<11.6$, this force is repulsive at distances up to $\sim 3 \mathrm{~nm}$, and attractive only upon contact. This is consistent with the usual DLVO description of interparticle forces: the surfaces are negatively charged, surrounded by clouds of $\mathrm{Ca}^{2+}$ ions, the overlap of which produces the measured repulsions. Adhesion upon contact is caused by Van der Waals forces only. In solutions of higher $\mathrm{pH}$, the force is attractive at all distances up to $\sim 3 \mathrm{~nm}$. 
The appearance of an attractive force at extremely high $\mathrm{pH}$ is an important phenomenon with respect to the cohesion of cement paste, which cannot be explained by a chemical binding of $\mathrm{Ca}^{2+}$ to the surfaces. Indeed, the formation of $\mathrm{Ca}^{2+}$ bridges would occur only near the isoelectric point of the surfaces ( $\mathrm{pH} \sim 11.6)$, and it would not give a force at distances as large as $3 \mathrm{~nm}$. At higher $\mathrm{pH}$, further binding of $\mathrm{Ca}^{2+}$ would result in a strong positive surface charge density, compensated by clouds of negatively charged ions. In a mean field model, the overlap of these clouds would again produce a repulsive force at intermediate distance.

These conflicts demonstrate that we do not have a good enough description of how C-S-H surfaces behave in a solution containing high concentrations of $\mathrm{OH}^{-}$and $\mathrm{Ca}^{2+}$. The failure of the " $\mathrm{Ca}^{2+}$ bridge" model casts a doubt on the hypothesis of a chemical binding of $\mathrm{Ca}^{2+}$ ions to the surfaces. The failure of the "ionic clouds" model (mean field) is not surprising, since such an approach is known to fail in conditions of high surface charge density and high ionic concentrations, especially so when the counterions are multivalent $[7,8]$.

\section{$\underline{\text { A better description of ionic interactions in cement paste }}$}

The dielectric continuum model where the ionic interactions are treated explicitly, and the water molecules are only incorporated via a dielectric permittivity, is probably sufficient for our purposes. However, we cannot use a mean field approximation, as is done in the PoissonBoltzmann equation; instead, the model must be solved exactly. This can be accomplished with, for instance, Monte Carlo (MC) simulations, where ion-ion correlations are taken into account $[7,8]$. For instance, an excess of positive charge at one location, near the surface of a particle, may be correlated with a lack of positive charge at the corresponding location near the opposing surface. It has already been demonstrated that such correlations can give rise to attractive forces [7-13].

Delville et al. have used MC simulations to calculate numerically the forces between flat parallel particles, in ionic conditions similar to those of cement paste $[12,13]$. They did find a strong attraction at high surface charge density. This was a major step in our understanding of interactions in cement paste. However, the attraction was found only at very short separations ( $0.4 \mathrm{~nm}$, corresponding to distances between silicate layers within a C-S-H particle rather than distances between particles) and in narrow conditions for the surface charge density. Such a strong dependence on molecular details is undesirable, because these details are not accurately known. Moreover, the predicted attraction does not match the AFM results, which indicate an attraction at intermediate distances, over a broad range of conditions. 
In the present work, we try to set up a model which is as simple and general as possible, without invoking unnecessary hypotheses, such as a dehydration of ions bound to surfaces, or, to the contrary, a fixed hydrated ion radius. Thus, we describe the interstitial solution with pure Coulomb interactions between all ions and between them and the surface charges, and we calculate the resulting ionic distributions, osmotic pressures, as well as the compensation of surface charges by counterions. The requirements on this model are that its predictions ought to be stable (i.e. no dramatic changes) with respect to small changes in surface charge density (x 2), surface-surface distances $( \pm 0.5 \mathrm{~nm})$ and dielectric constant of the solution (down to $\varepsilon_{\mathrm{r}}=50$ ). Finally, the predictions of the model are compared with the experimental results, i.e. the reversal of the apparent particle charge and of the interparticle force measured at intermediate distances.

\section{Surface Chemistry of C-S-H}

The C-S-H particles are made of silicate layers separated by interlayer spaces containing calcium, oxygen and a few water molecules [14]. The thickness of the interlayer spaces is about $0.4 \mathrm{~nm}$, and that of the silicate layers is $1 \mathrm{~nm}$. A C-S-H particle contains, on the average, 4 silicate layers and 3 interlayer spaces, giving a thickness of $5 \mathrm{~nm}$.

\section{Surface sites}

The outer surfaces of the C-S-H nanoparticles are made of silicate, and they have $\mathrm{SiOH}$ surface groups. The surface charge density may be estimated from the structure [15] of C-S-H in which three tetrahedra bearing two silanols occupy $0.412 \mathrm{~nm}^{2}$, i.e. 4.85 sites $/ \mathrm{nm}^{2}$. At high $\mathrm{pH}$, these silanols react with $\mathrm{OH}^{-}$ions of the solution to give $\mathrm{SiO}^{-}$groups. From the evolution of stoichiometry of C-S-H dispersions in calcium hydroxide solutions, it is deduced that, at $\mathrm{pH}$ values below 11 , the density of negative $\mathrm{SiO}^{-}$groups is low, and comparable to the usual surface charge densities of colloidal dispersions; when $\mathrm{pH}$ is increased from 11 to 12 , it rises quite fast, and beyond $\mathrm{pH}=12$ it saturates at a high value, corresponding to nearly complete ionization of all surface sites [14].

Apparent surface charge

The surface charges of the C-S-H nanoparticles are compensated by $\mathrm{Ca}^{2+}$ counterions in the solution. Some of these counterions are localized close to $\mathrm{SiO}^{-}$surface sites, and therefore reduce the apparent negative surface charge of the C-S-H particles. In electrophoretic experiments, it has been found that the release of $\mathrm{Ca}^{2+}$ in the solution and the corresponding 
rise in $\mathrm{pH}$ cause a reversal in the mobility of the particles: at low $\mathrm{Ca}^{2+}$ and $\mathrm{OH}^{-}$concentrations they move as negatively charged particles, and at high $\mathrm{Ca}^{2+}$ and $\mathrm{OH}^{-}$concentrations as positively charged particles $[5,14]$. The inversion point is located in the vicinity of $\mathrm{pH}=11.6$, corresponding to $\left[\mathrm{OH}^{-}\right]=4.10^{-3} \mathrm{M}$ and $\mathrm{Ca}^{2+}=2 \cdot 10^{-3} \mathrm{M}$. The charge reversal is most likely a consequence of the interaction between the anionic $\mathrm{SiO}^{-}$groups o the particles and the $\mathrm{Ca}^{2+}$ ions in the solution. In a mean field Gouy-Chapman model, there is a strong accumulation of counterions close to the charged surface but this never leads to a charge reversal. To account for the observation, one has to go beyond the mean field electrostatic model.

One possibility is to invoke an additional, short ranged non-Coulombic interaction between surface charges and counterions. In chemical terms this could involve the formation of an inner-sphere complex, where the $\mathrm{Ca}^{2+}$ loses some of its hydration water and makes a direct ionic bond to the $\mathrm{SiO}^{-}$surface site through the reaction:

$$
\mathrm{SiO}^{-}---\mathrm{HOH}+\mathrm{HOH}---\mathrm{Ca}^{2+} \rightarrow \mathrm{SiOCa}^{+}+2 \mathrm{HOH}
$$

Such bonds exist within the C-S-H particles, where they connect the silicate layers to the $\mathrm{Ca}^{2+}$ ions in the interlayer spaces. They must also be formed in conditions where the particles are growing, collecting ions form the solution.

Another alternative is to consider the formation of a solvent separated ion coordination of the type:

$$
\mathrm{SiO}^{-}---\mathrm{HOH}+\mathrm{HOH}---\mathrm{Ca}^{2+} \rightarrow \mathrm{SiO}^{-}---\mathrm{HOH}---\mathrm{Ca}^{2+}+\mathrm{HOH}
$$

To account for a charge reversal by either of these two schemes, the short range attractive term would have to be strong enough to provide binding even without being boosted by the long range electrostatic interaction. The general experience is that cations do not interact with a strong specificity with negatively charged surfaces [16]; however, we are not in a position to unequivocally exclude the possibility of charge reversal by specific binding effects.

Instead, we will pursue an alternative explanation of the observations, based on a purely electrostatic mechanism that goes beyond the mean field Gouy-Chapman description and includes ion-ion correlation effects. Furthermore in this way we can simultaneously account for both electrophoretic charge reversal and attractive interparticle forces in systems of high surface charges. 


\section{$\underline{\text { Ionic concentrations }}$}

At the $\mathrm{pH}$ value where the charge reversal occurs, and the interparticle force becomes attractive, the bulk ionic concentrations are not high: $\left[\mathrm{OH}^{-}\right]=4 \cdot 10^{-3} \mathrm{M}$ and $\left[\mathrm{Ca}^{2+}\right]=2 \cdot 10^{-3}$ M. The average separation of $\mathrm{Ca}^{2+}$ ions is on the order of $10 \mathrm{~nm}$, and their electrostatic interaction energy is much below thermal energies. More quantitatively, the Debye screening length $\kappa^{-1}$, which gives the range of electrostatic interactions in the solution, is on the order of $4 \mathrm{~nm}$ at this $\mathrm{pH}$. A commonly used criterion is that the solution may precipitate when the Debye length becomes shorter than the Bjerrum length: this is clearly not the case in the conditions detailed above (in fact the precipitation threshold is at $\left[\mathrm{Ca}^{2+}\right]=20 \cdot 10^{-3}$, in agreement with this criterion).

On the other hand, the concentrations of $\mathrm{Ca}^{2+}$ ions in the vicinity of C-S-H surfaces are extremely high, because of the high surface charge density. The region of electrostatic confinement has a thickness given by the Gouy-Chapman length, $l_{G C}$, which is:

$$
l_{G C}=\frac{e}{2 \pi z \sigma l_{B}}
$$

where $e$ is the elementary charge, $\mathrm{z}$ the counterion valency, $\sigma$ the surface charge density and $l_{B}$ the Bjerrum length. For C-S-H particles in solutions of very high $\mathrm{pH}(\mathrm{pH}=11.6-12.6)$, the surface density of ionized sites is in the range $2.5-5 \mathrm{~nm}^{-2}, \sigma$ in the range $0.4-0.8 \mathrm{C} / \mathrm{m}^{2}$ and $l_{G C}$ of the order of $0.1 \mathrm{~nm}$; thus, most counterions are confined in the immediate vicinity of the surfaces. In the space that separates two approaching C-S-H particles, these confined counterions are much more numerous than the ions from the bulk electrolyte. For instance, if two C-S-H particles separated by $2 \mathrm{~nm}$, the formal concentration of $\mathrm{Ca}^{2+}$ ions that are brought by the particles is $2 \mathrm{M}$, which is 1000 times higher than the bulk concentration.

\section{The model}

We use the primitive model where all ions are considered explicitly and water is treated as a dielectric continuum, characterized by its dielectric permittivity $\varepsilon_{r}$. The calculations can be performed with the bulk value $\varepsilon_{r}=78,5$, or with a lower value if conditions make it necessary. Another simplification is that the charge on the C-S-H nanoparticles is treated as uniformly smeared out. Furthermore, it is assumed that the interaction between C-S-H nanoparticles can be modeled as an interaction between two parallel planar walls, with a smeared surface charge 
density $\sigma$. An appropriate number of counterions, $\mathrm{Ca}^{2+}$, and coions, $\mathrm{OH}^{-}$, are dissolved in the intervening space and make the total system electroneutral (Figure 1).

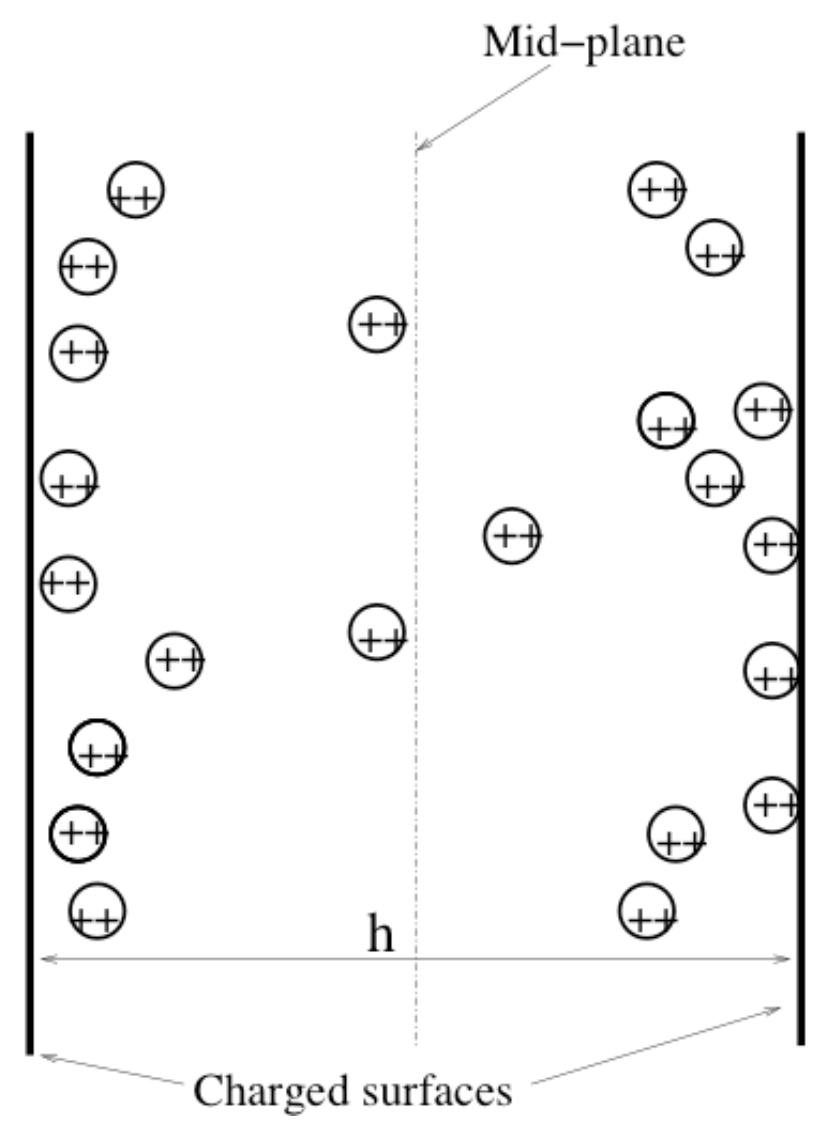

Figure 1. Schematic representation of two walls with negatively charged $\mathrm{SiO}^{-}$groups and neutralizing $\mathrm{Ca}^{++}$counterions. Additional salt pairs have been left out for clarity. $\underline{\text { Ionic interactions }}$

The interaction energy between any two ions of radius $a$ can be formally described by:

$$
\begin{array}{ll}
u\left(\mathbf{r}_{i}, \mathbf{r}_{j}\right)=\frac{q_{i} q_{j} e^{2}}{4 \pi \varepsilon_{0} \varepsilon_{r}\left(\mathbf{r}_{i}-\mathbf{r}_{j}\right)} & r>a \\
u\left(\mathbf{r}_{i}, \mathbf{r}_{j}\right)=\infty & r<a
\end{array}
$$

where $e$ and $\varepsilon_{0}$ are the elementary charge and electric permittivity for vacuum, respectively. The hard core diameter $a$ has a clear physical origin, that is, ions cannot overlap due to the quantum mechanical exchange repulsion. In the present study, it is set equal to $0.4 \mathrm{~nm}$. The exact value of $a$ is of course not known, but fortunately for the present study of cement paste, there is a broad range of $a$ where it has no effect on the interparticle forces. That is, the Coulomb hole that surrounds an ion is larger than the hard core diameter. This "safe" situation 
is found in simulations with only counterions present in the interstitial volume, at all surface charge densities except for the very highest one. Thus, we could in principle have set the counterion diameter to zero without changing the results.

The counterions and any added salt are interacting with each other and with the charged surfaces. At the surfaces, we allow the centers of the ions to come right up to the confining surface. Thus we do not make any assumption concerning the hydration of ions that are in contact with the surfaces, except that their interaction energy is purely electrostatic. This means that the hard core radius has no effect on the ion-wall interaction whatsoever. If on the other hand we only allow the ions to touch the surface it only means that the separation $h$ takes another value, and all our calculated forces curves are just shifted by $0.4 \mathrm{~nm}$.

These interactions are applied to all ions in a "box" defined by the charged walls (separation $h$, lateral dimensions chosen such that the box contains a set number of counterions). However, electrostatic interactions are long ranged, and a correction term approximating interactions outside the Monte Carlo box is also added [17]. Thus, the total energy is a sum of ion-ion, ion-wall, wall-wall interactions plus a long-range correction term:

$$
U_{t o t}=U_{i i}+U_{i w}+U_{w w}+U_{l r}
$$

\section{Monte Carlo simulations}

The electrostatic interaction described above defines the Hamiltonian, i.e. forms the basis for a MC simulation of a cement paste. We use the standard Metropolis algorithm [18] in the canonical ensemble. In the majority of the simulations, 180 counterions were included, but test simulations with twice this number were performed to ensure size convergence. Each particle was subject to $10^{5}$ attempted moves. Simulations with $10^{6}$ moves/ion confirmed that convergence was achieved with respect to number of configurations. The results are the equilibrium distributions of all ions in the interstitial solution and the direct force acting between the two halves of the system shown in Figure 1. From this knowledge we calculate the osmotic pressure.

\section{$\underline{\text { Osmotic pressure }}$}

The excess osmotic pressure of the interstitial solution may be calculated from the ionic distributions according to either of the two following expressions [7]:

$$
p_{o s m}=k_{B} T \sum_{i} c_{i}(\text { wall })-\frac{\sigma^{2}}{2 \varepsilon_{r} \varepsilon_{0}}
$$




$$
p_{o s m}=k_{B} T \sum_{i}\left[c_{i}(m p)+p_{i}^{c o r r}+p_{i}^{h c}\right]
$$

In these expressions, the pressures $k_{B} T c_{i}($ wall $)$ and $k_{B} T c_{i}(m p)$ are calculated from the concentrations of ions of type $i$, respectively near a charged wall and at the mid-plane of the cell. The Maxwell term $\sigma / 2 \varepsilon_{r} \varepsilon_{0}$ is the sum of interactions of a wall with all ions in the box and with the other charged wall. The term $p_{i}^{\text {corr }}$ is also electrostatic in origin: it comes from the fact that ions on either side of the mid-plane correlate. In the mean field description this term disappears due to electroneutrality, i.e. there would be no correlations across the midplane. In real life, however, this term gives an attractive contribution to the pressure, which can overcome the entropic repulsive term. Finally, if the ions have a finite size $a$, one also gets a hard core term, $p_{i}^{h c}$, which describes the additional pressure due to the finite volume of the ions. At most ionic concentrations, this term is quite small, and can be ignored without loss of physical significance. However, at the highest ionic concentration, it may become significant, and compensate the attraction due to ion-ion correlations [19].

These relations are exact within the primitive model. Here we have used the second formulation for the evaluation of the pressure, since it tends to give the best accuracy.

\section{Results}

The main results of the MC calculations are the magnitudes of the three contributions to the excess osmotic pressure of the solution that separates C-S-H particles: entropic, correlation and hard core (see equation /5/). In this section, we present the variations of these pressures with the surface charge density $\sigma$. Since $\sigma$ is determined by $\mathrm{pH}$, these variations make it possible to predict whether or not the total pressure will change from repulsive to attractive during cement hydration. Next, other MC calculations are presented in order to determine how the surface charge of isolated particles is compensated by counterions. From these we can predict whether the apparent surface charge will change from negative to positive during early cement hydration.

\section{Entropic pressure}

This repulsive force originates from the "gas" pressure of the counterions. Its magnitude is proportional to the concentration of counterions at the mid-plane of the cell depicted in Figure 1 , according to equation $/ 6 /$. As the surface charge density rises, the number of counterions 
increases, but they become increasingly accumulated at the surfaces. Figure 2 shows the net result: the entropic pressure decreases dramatically at high surface charge densities. This is in contrast to the mean field result, where the pressure increases asymptotically. The variation is essentially the same at all separations, but the absolute magnitude of the pressures is larger at shorter separations.

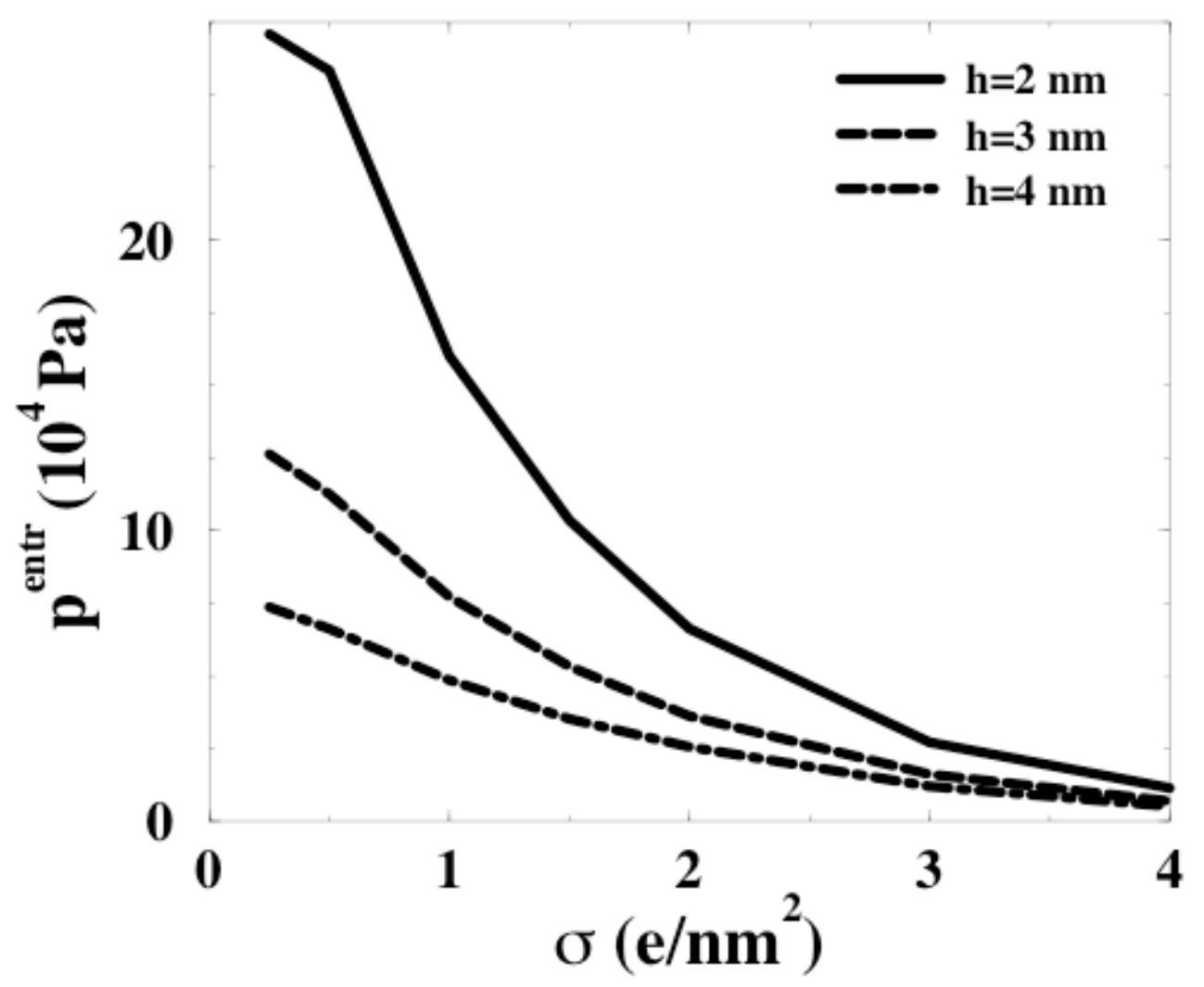

Figure 2. Entropic pressure of the divalent counterions, as a function of the surface charge density $\sigma$. This entropic pressure is calculated from the concentration of counterions at the mid-plane of the cell depicted in Figure 1, according to equation /6/. The three curves correspond to cells with different separations $h$ between surfaces (see Figure 1): $h=2 \mathrm{~nm}$ (higher curve), $h=3 \mathrm{~nm}$ (middle) and $h=4 \mathrm{~nm}$ (lower). All three curves show a collapse of the entropic pressure at high surface charge density. Note that the high surface charge densities originate from extremely high $\mathrm{pH}$ values $(11.6-12.6)$

\section{Correlation pressure}

This attractive force is caused by correlations between counterions on either side of the midplane, i.e. an excess of counterions on one side is correlated with a lack of counterions on the opposite side, causing an attraction. Figure 3 shows how the attractive pressure depends on 
the surface charge density $\sigma$. It is found that the magnitude of the correlation effect decreases with increasing $\sigma$, although its relative importance increases (see below). The reduction of the magnitude comes from the fact that high surface charge densities lead to compact layers of counterions at each surface. However, the reduction is rather modest: the pressure decays by a factor of 2 when $\sigma$ rises from 0.5 to $2 \mathrm{e} / \mathrm{nm}^{2}$, and at higher $\sigma$ it then remains approximately constant. Note that the correlation term, $p^{\text {corr }}$, decays asymptotically as $h^{-3}$ [20], which is in fair agreement with the three curves in Figure 3 at the highest surface charge density (their ratio should be 64:27:8).

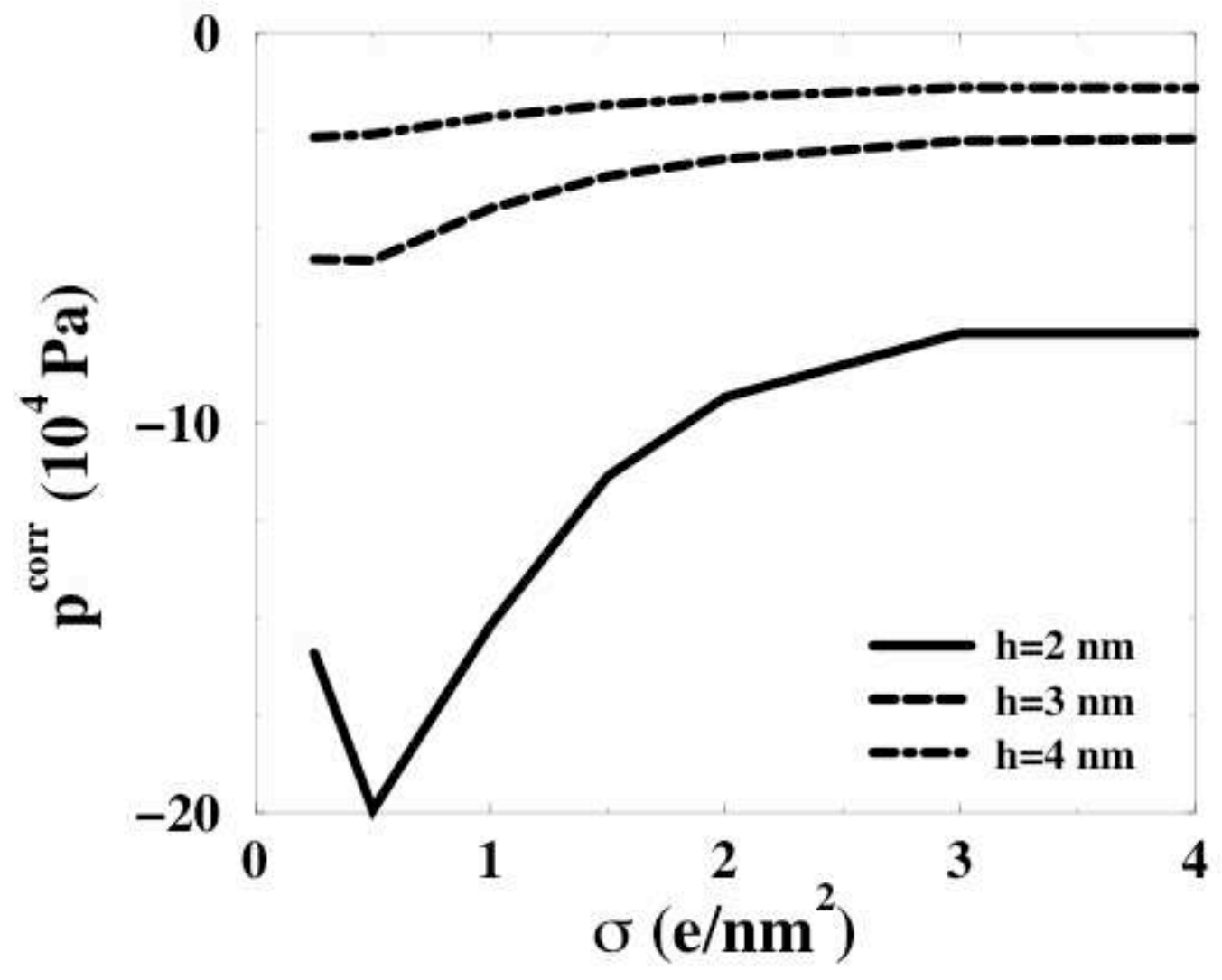

Figure 3. Correlation pressure of the divalent counterions, as a function of the surface charge density $\sigma$. This term is attractive (the pressures are negative), and it is much stronger at short surface - surface separations $(h=2 \mathrm{~nm}$, lower curve) than at larger separations $(h=3$ $\mathrm{nm}$ and $4 \mathrm{~nm}$, upper curves).

Total osmotic pressure

The total osmotic pressure is the sum of the entropic, correlation and hard-core terms. As explained above, we found that the hard-core term was insignificant at all realistic surface - 
surface separations. At low surface charge densities, the entropic pressure collapses, and the total pressure turns attractive (Figure 4). This reversal occurs earlier (i.e. at lower $\sigma$ ) when the surfaces are closer together: indeed, the attractive correlations are stronger in such conditions (Figure 3).

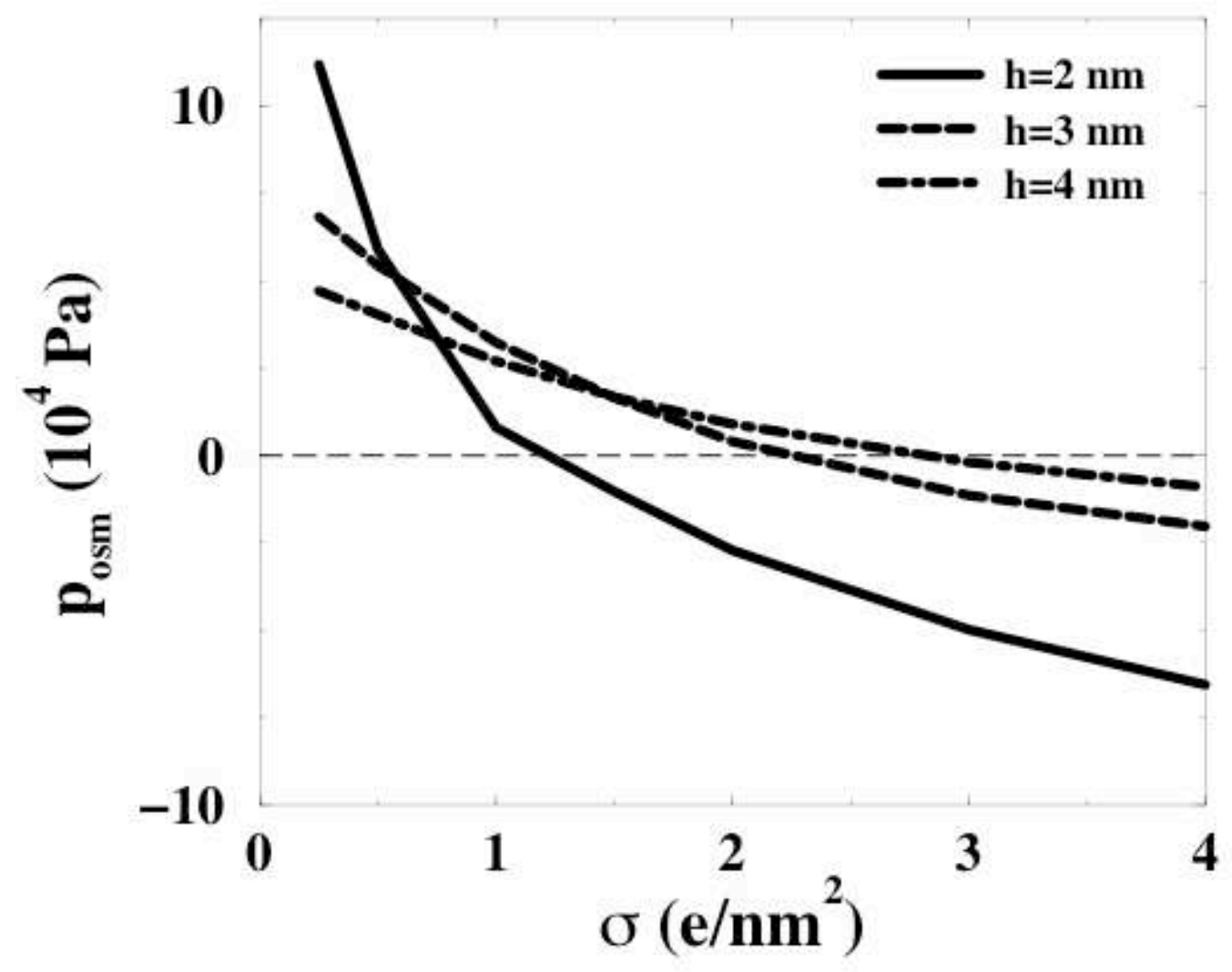

Figure 4. Total osmotic pressure due to counterions in the interstitial solution that separates two opposing C-S-H surfaces, as a function of surface charge density $\sigma$. The three curves correspond to different separations $h$ between surfaces (see Figure 1): $h=2 \mathrm{~nm}$ (full line), $h=3 \mathrm{~nm}$ (dashed line) and $h=4 \mathrm{~nm}$ (dashed-dotted line). In each case, the pressure changes from repulsive (no cohesion) to attractive (onset of cohesion) as the surface charge density becomes sufficiently high. The change occurs at surface charge densities $\sigma=1$ to 3 $\mathrm{e} / \mathrm{nm}^{2}$, which corresponds to $\mathrm{pH}$ values in the range 11 to 11.7 according to surface ionization equilibria.

Another way to view the changes caused by the rise in $\sigma$ is to examine the pressure / separation curves at chosen values of $\sigma$ (Figure 5). At low surface charge densities, these curves show a monotonic repulsion; at intermediate values of $\sigma$, a weak long range repulsion 
gives way to a strong short range attraction; finally, at high surface charge densities, the curves are attractive for the whole range of separations. These results demonstrate that the rise in $\mathrm{pH}$, and the corresponding rise in $\sigma$, cause $\mathrm{C}-\mathrm{S}-\mathrm{H}$ particles to attract each other and bind at contact.

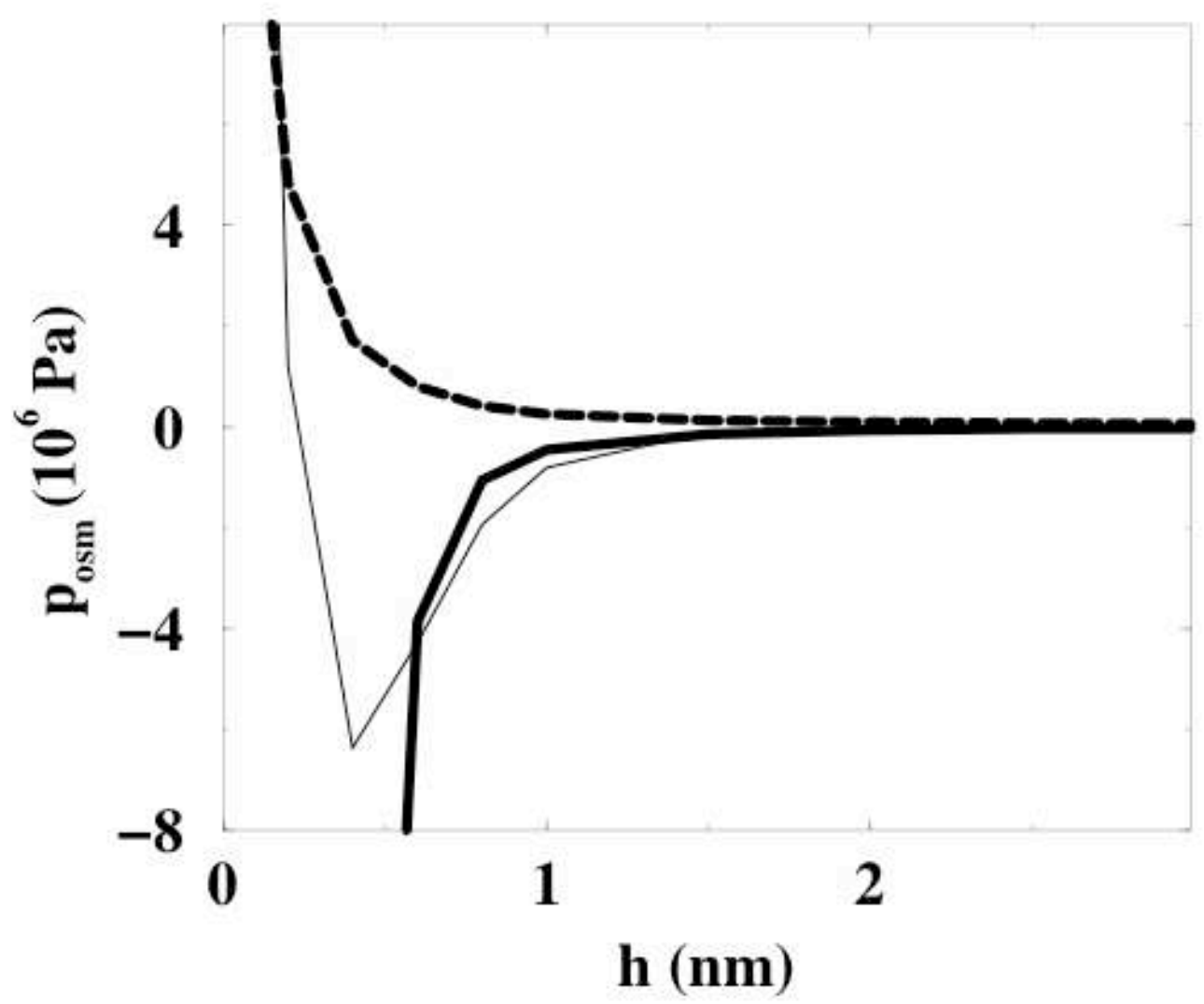

Figure 5. Total osmotic pressure due to counterions, as a function of surface - surface separation $h$. The three curves correspond to increasing values of the surface charge density: $\sigma$ $=0.3$ (dashed line), 1.25 (thin solid line) and $5 \mathrm{e} / \mathrm{nm}^{2}$ (thick solid line).

\section{$\underline{\text { AFM Force }}$}

The geometry of the AFM tip is not easily known, but we may for convenience assume that the relevant part is approximately planar. This is of course a very rough approximation to the configuration of C-S-H surfaces in the AFM experiments reported by Lesko et al. [6], where C-S-H surfaces were created on an AFM tip and on the opposite plane, but should still suffice for a qualitative comparison.

The total interparticle force is the sum of the ionic contribution (net osmotic pressure) and the Van der Waals force. Figure 6 shows the ionic contribution alone for a tip area equal to 5000 $\mathrm{nm}^{2}$. At very low surface charge densities, the force curve has a repulsive barrier, followed at 
shorter separation by an attractive well. At high surface charge densities, the force curve is attractive at all separations. This is in good agreement with the measurements published by Lesko et al [6], and with more recent results obtained by Plassard et al. (Figure 7).

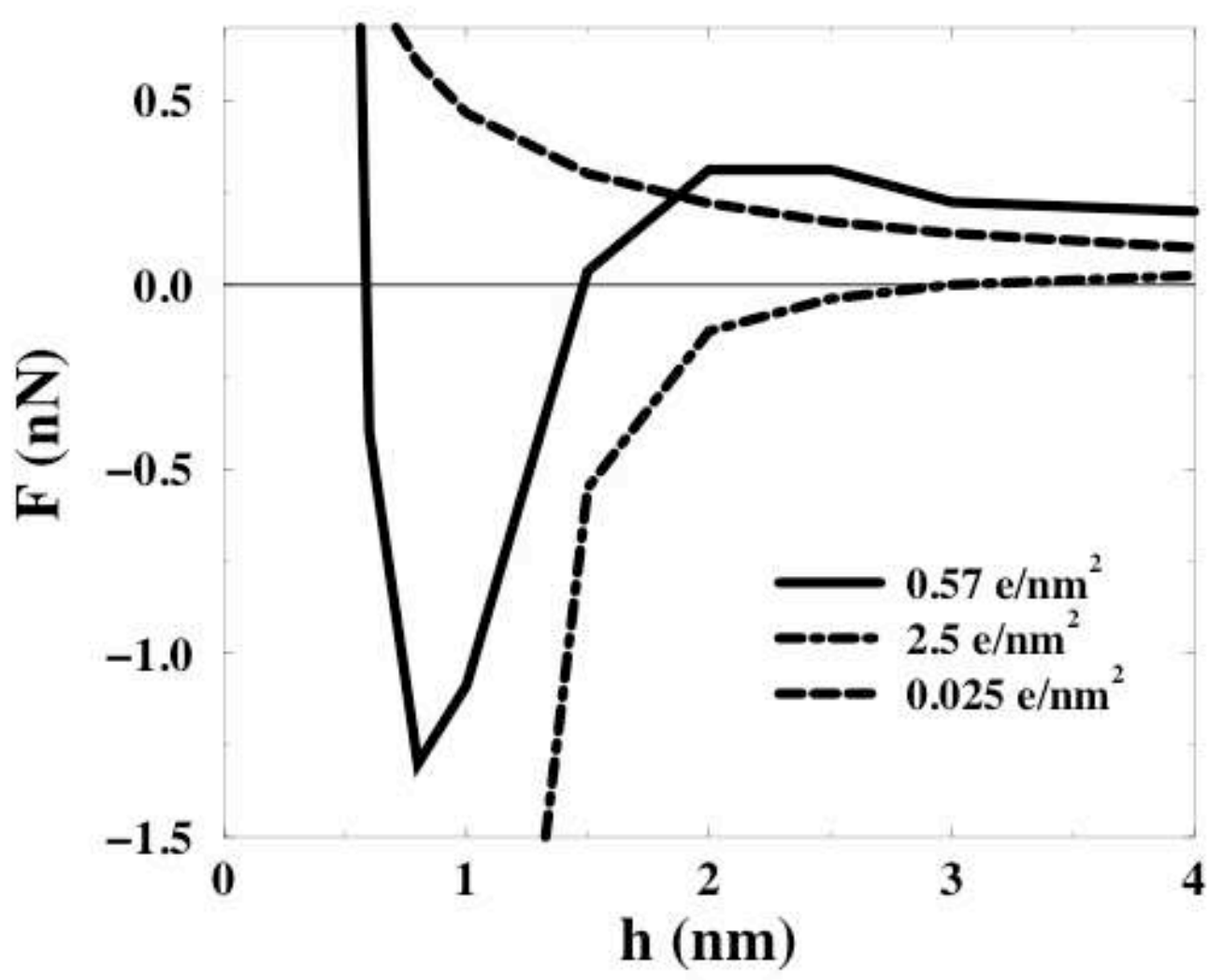

Figure 6. Predicted force vs. separation for AFM experiments. The force includes the osmotic pressure of the counterions (cf. Figure 5) but not the Van der Waals attraction (see the text). The upper curve with a low $\sigma$ corresponds to a low $\mathrm{pH}(<10)$; in these conditions the entropic pressure of the counterions is the strongest term. The second curve with $\sigma=0.57$ $\mathrm{e} / \mathrm{nm}^{2}$ corresponds to conditions where the entropic pressure is depressed; under these conditions, the AFM experiments show a weak repulsive barrier followed by an adhesion at contact (see the next figure). The lower curve corresponds to a high surface charge density $\sigma$ $=2.5 \mathrm{e} / \mathrm{nm}^{2}$ that causes the entropic pressure to collapse completely; under these conditions, the AFM experiments show an attractive force all the way to contact, as predicted. 


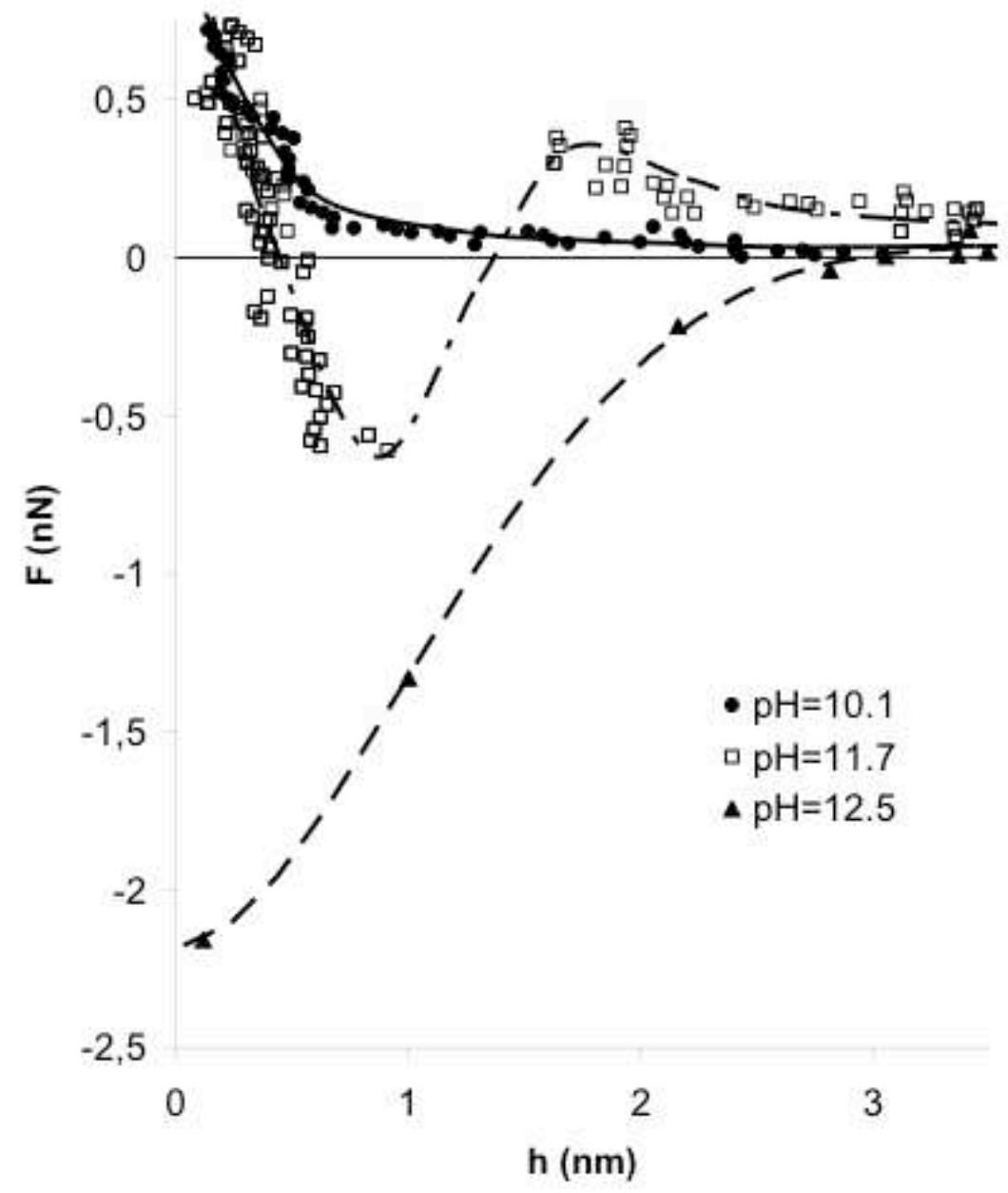

Figure 7 Experimental force curves obtained through AFM by Plassard et al. (to be published).

For a quantitative comparison, the contribution of the Van der Waals attraction should be included in the calculated force. This may be calculated as:

$F_{v d W}=-\frac{A}{6 \pi\left(h_{v d W}\right)^{3}}$

where $\mathrm{A}$ is the Hamaker constant for the $\mathrm{CSH}$ - water system, and $h_{v d W}$ is the distance between the planes that mark the discontinuity in polarizability between the ionic solution and the solid CSH. The Hamaker constant for C-S-H is not known, but the Hamaker constant for amorphous silica is $0.46 \cdot 10^{-20} \mathrm{~J}$, and that for mica is $1.34 \cdot 10^{-20} \mathrm{~J}$; hence we may take $\mathrm{A}=$ $10^{-20} \mathrm{~J}$ for the Hamaker constant of C-S-H. On the other hand, the precise value of $h_{v d W}$ is not known. A realistic choice would be $h_{v d W}=h+1 \mathrm{~nm}$, and then the Van der Waals contribution is small and does not modify the qualitative features of the AFM forces shown in Figure 6. 
The opposite choice would be $h_{v d W}=h$ (unrealistic because the polar groups of the CSH surfaces are hydrated), and then all curves shown in Figure 6 become attractive. Thus, the good agreement between the curves calculated for the ionic contribution alone (Figure 6) and the experimental curves (Figure 7) indicates that the van der Waals separation $h_{v d W}$ is significantly larger than the ionic separation $\mathrm{h}$, which is expected for ionic surfaces.

\section{Apparent surface charge}

When particles are dispersed in a solution containing some passive electrolyte, their surfaces can accumulate counterions from the electrolyte in addition to their own counterions. With divalent counterions, the accumulated ionic charge may overcompensate the surface charge, and the particles appear in electrokinetic experiments as having reversed their charge [21-23].

This effect can be studied through MC calculations, which determine the distribution of counterions and coions in the vicinity of surfaces. Figure 8 shows that, at high surface charge densities, the $\mathrm{Ca}^{++}$counterions are accumulated right next to the surfaces, while the co-ions from the electrolyte are excluded from that region. The amount of accumulated counterions overcompensates the surface charge, and at larger distances (about $0.5 \mathrm{~nm}$ ) this apparent positive charge is compensated by a concentration of coions that shows a non-monotonic profile.

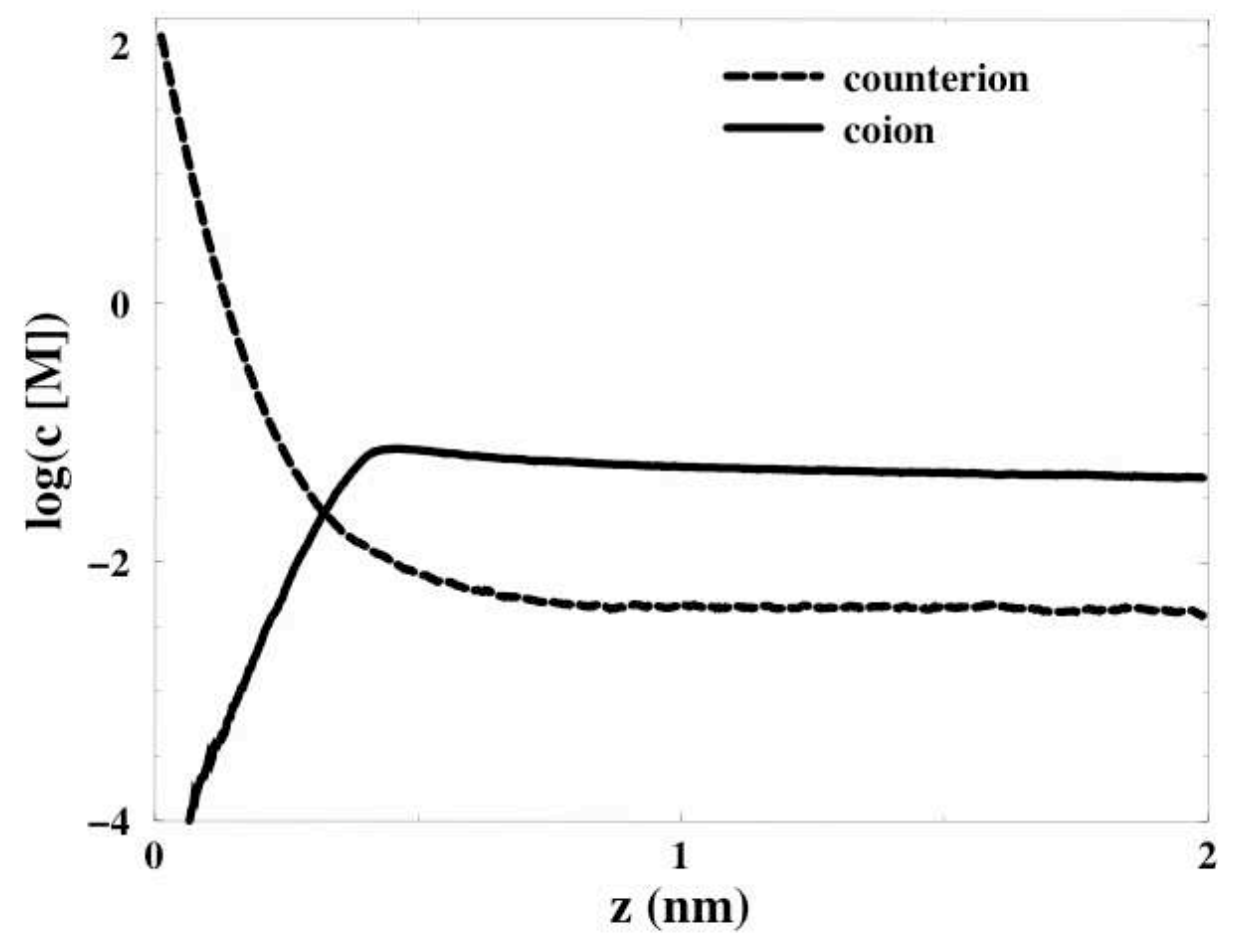


Figure 8. The simulated distribution of coions (solid line) and counterions (dashed line) outside a charged surface $\left(\sigma=5 \mathrm{e} / \mathrm{nm}^{2}\right)$ located at $z=0$. The average density of divalent counterions $\left(\mathrm{Ca}^{2+}\right)$ and monovalent coions $\left(\mathrm{OH}^{-}\right)$is $2.08 \mathrm{M}$ and $0.046 \mathrm{M}$, respectively.

The extent of overcompensation can be expressed as the total charge accumulated from the surface up to a plane located at a distance $z$ from the surface (including the surface charges and all counterions located next to the surface, up to the distance $z$ ). An example of overcompensation is shown in Figure 9, where the accumulated charge is calculated as a function of the distance $z$ to the surface, for various values of the surface charge density $\sigma$. At low surface charge densities, the number of condensed counterions is not enough to compensate the surface charges, and so the total accumulated charge remains negative up to the mid-plane. At high surface charge densities, however, extensive accumulation takes place, to the extent that the surface charge is overcompensated. Then, at all distances $z>2 \mathrm{~nm}$ the particle appears as if it were positively charged, and surrounded by negative ions from the added electrolyte.

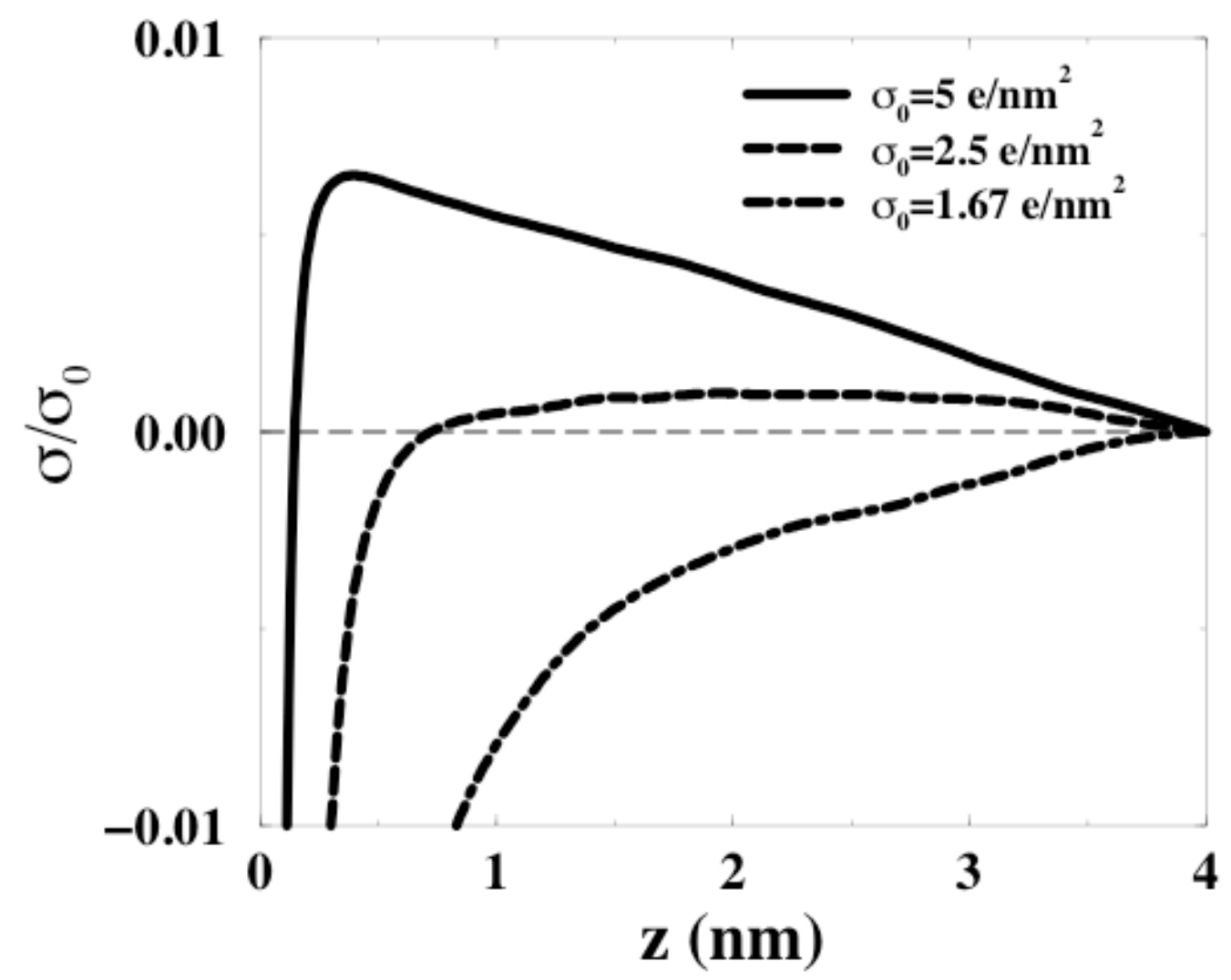


Figure 9. Overcompensation of the surface charge by $\mathrm{Ca}^{2+}$ counterions, in a dispersion containing a small amount of 2:1 electrolyte. The curves indicate the total accumulated charge, including the surface charges, the counterions and the co-ions, up to a distance $z$ from the surface. The calculations are made for a cell with a surface - surface separation $h=8 \mathrm{~nm}$. Each curve corresponds to a set surface charge density: $\sigma=1.67 \mathrm{e} / \mathrm{nm}^{2}$ (lower curve, the surface charge is undercompensated), $\sigma=2.5 \mathrm{e} / \mathrm{nm}^{2}$ (middle curve, slight overcompensation), $\sigma=5 \mathrm{e} / \mathrm{nm}^{2}$ (upper curve, strong overcompensation).

The extent of overcompensation depends on the availability of added salt, $\mathrm{Ca}(\mathrm{OH})_{2}$ : Figure 10 shows that the accumulated charge increases with the amount of added $\mathrm{Ca}(\mathrm{OH})_{2}$. It is also interesting to note that this strong overcharging takes place close to the surface, hence it remains essentially the same regardless of the separation of the surfaces $(2-16 \mathrm{~nm})$. Consequently, this result applies to dilute dispersions as well.

The accumulated charge is predicted to become positive when the surface charge density $\sigma$ exceeds $2 \mathrm{e} / \mathrm{nm}^{2}$, which must occur at high $\mathrm{pH}$. Indeed, electrokinetic experiments [5] do show a reversal of the sign of the mobility when the $\mathrm{pH}$ of the dispersion exceeds 11.5. Thus, the reversal of the apparent charge is explained and reproduced through plain electrostatic accumulation of $\mathrm{Ca}^{2+}$ counterions. 


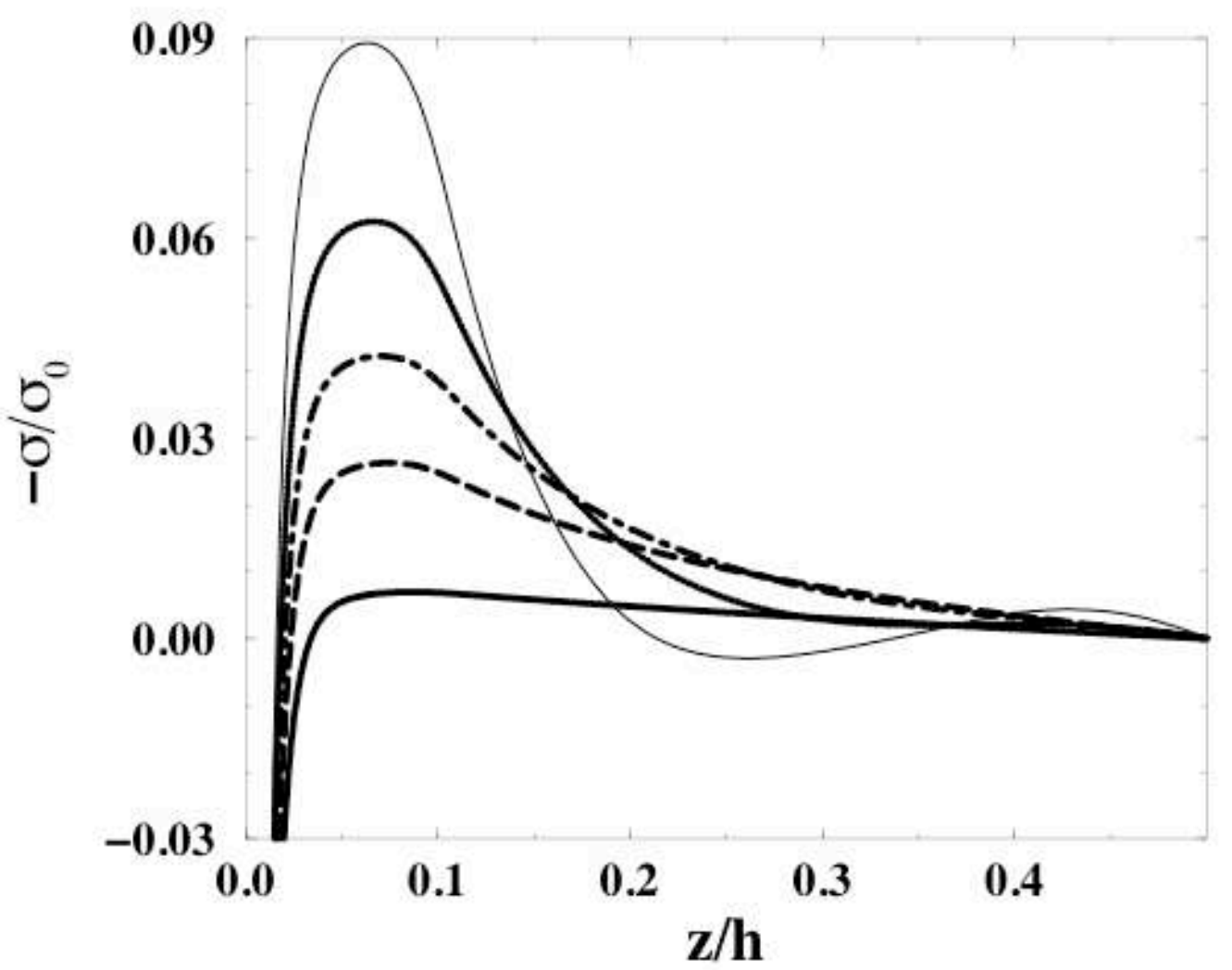

Figure 10. Overcompensation of the surface charge in dispersions with different amounts of salt: solid line $=0.023$, dashed line $=0.092$, dot-dashed line $=0.18$, dotted line $=0.369$, and thin solid line $=0.738 \mathrm{M}$ of a fictitious $2: 1$ electrolyte. The surface - surface separation is $h=4 \mathrm{~nm}$ in each case. The strong overcompensation observed at high salt is due to the accumulation of $\mathrm{Ca}^{2+}$ ions from the salt onto the surface; it takes place close to the surface, so that the surface appears as positively charged in experiments that sample larger distances to the surface.

\section{Conclusions}

The interactions of C-S-H particles with small ions in the interstitial solution of cement paste can be described through a model where the sole ingredient is the Coulomb interactions between all electrical charges. This model makes two main predictions, regarding the apparent charges of particles in dilute dispersions, and the interactions of particles in concentrated dispersions.

The apparent charge of C-S-H particles results from surface charges $\left(\mathrm{SiO}^{-}\right)$plus the charges of divalent $\mathrm{Ca}^{2+}$ counterions that are accumulated at the surfaces. In dilute dispersions, and at 
high surface charge density, the model shows that the accumulation of $\mathrm{Ca}^{2+}$ at the C-S-H surfaces overcompensates the surface charges. Therefore the reversal of the apparent particle charge is a consequence of their very high surface charge density (i.e. high $\mathrm{pH}$ ), and does not require a chemical binding of the $\mathrm{Ca}^{2+}$ ions to the surfaces.

The interactions between particles result from the competition of an entropic pressure (proportional to the concentration of counterions at the mid-plane in between two surfaces) with an attractive force (due to the correlations of counterions located on either side of the midplane). One important parameter that rules the relative magnitudes of these forces is the surface charge density. At high surface charge densities, the entropic pressure collapses, due to the accumulation of counterions at the surfaces, and the attractive force dominates the interactions. Therefore the medium-range attraction between $\mathrm{C}-\mathrm{S}-\mathrm{H}$ particles, which has been measured in AFM experiments, is a consequence of their very high surface charge density, and the presence of divalent counterions. An increased surface charge density leads to a stronger but more short range attraction.

The existence of medium-range attractions between particles may have important consequences for the formation of the C-S-H network. During the early stages of growth, when "branches" of C-S-H aggregates grow on neighboring C3S grains, short range forces may help neighboring branches to connect to each other. At later stages, short range attractive forces may control the maximum strain of the C-S-H network.

The same model could handle situations where other ions (monovalent or multivalent) are present, due to variations in the composition of the cement paste. In this respect, it is important to point out that the phenomena described here do not require a high bulk concentration of $\mathrm{Ca}^{2+}$ ions. Therefore similar phenomena can be expected whenever the $\mathrm{pH}$ is extremely high and at least some divalent ions are present. Moreover, attractive forces can also be found in presence of monovalent counterions only, but only at shorter ranges $(1 \mathrm{~nm})$ and therefore higher local ionic concentrations [24].

\section{References}

$1 \quad$ Nonat, A. Materials Structures 27, 187 (1994)

2 Jiang, S.P., Mutin, J.C., Nonat, A. Cement Concrete Res. 25, 779 (1995)

3 Nachbaur, L., Mutin, J.C., Nonat, A., Choplin, L. Cement Concrete Res. 31, 183 (2001) 
4 Nonat, A., Courault, A.C., Damidot, D. Submitted to "Journal of Colloid and Interface Science”

5 Nachbaur, L., Nkinamubanzi, P.-C., Nonat, A., Mutin, J.-C. J. Colloid Interface Sci. 202, $261(1998)$

6 Lesko, S., Lesniewska, E., Nonat, A., Mutin, J-C., Goudonnet, J-P. Ultramicroscopy $86,11(2001)$

7 Guldbrand, L., Jönsson, B., Wennerström, H., Linse, P. . J. Chem. Phys. 80, 2221 (1984)

8 Jönsson, B., Wennerström, H. In: Electrostatic Effects in Soft Matter and Biophysics Kluwer Academic publishers, Eds. C. Holm, P. Kekicheff, R. Podgornik (2001)

9 Svensson, B., Jönsson, B. Chem. Phys. Lett. 108, 580 (1984)

10 Kjellander, R., Marcelja, S. J. Chem. Phys. 82, 2122 (1985)

11 Valleau, J.P., Ivkov, R., Torrie, G.M. .J. Chem. Phys. 95, 520 (1991)

12 Pellenq, R., Caillot, J., Delville, A. J. Phys. Chem. B 101, 8584 (1997)

13 Delville, A., Pellenq, R., Caillol, J. J. Chem. Phys. 106, 7275 (1997)

14 Viallis-Terrisse, H., Nonat, A., Petit, J.-C. J. Colloid Interface Sci. 244, 58 (2001)

15 Taylor, H.W.W. in Cement Chemistry; Academic Press, London 1990; p 142

16 Wennerström, H., Khan, A., Lindman, B. Adv. Colloid Interface Sci. 34, 433 (1991)

17 Jönsson, B., Wennerström, H., Halle, B. J. Chem. Phys. 84, 2179 (1980)

18 Metropolis, N.A., Rosenbluth, A.W., Rosenbluth, M.N., Teller, A., Teller, E. J. Chem. Phys. 21, 1087 (1953)

19 Kjellander, R., Marcelja, S. J. Phys. (France) 49, 1009 (1988)

20 Attard, P., Mitchell, D.J., Ninham, B.W. J. Chem. Phys. 89, 4358 (1988)

21 Sjöstrom, L., Åkesson, T., Jönsson, B. Ber. Bunsenges. Phys. Chem. 100, 889 (1996)

22 Shklovskii, B.I. Phys. Rev. E 60, 5802 (1999)

23 Messina, R., Holm, C., Kremer, K. Euro. Phys. J. E 4, 363 (2001)

24 Sonneville-Aubrun, O., Bergeron, V., Gulik-Krzywicki, T., Jönsson, Bo, Wennerström, H., Lindner, P., Cabane, B. Langmuir 16, 1566 (2000) 


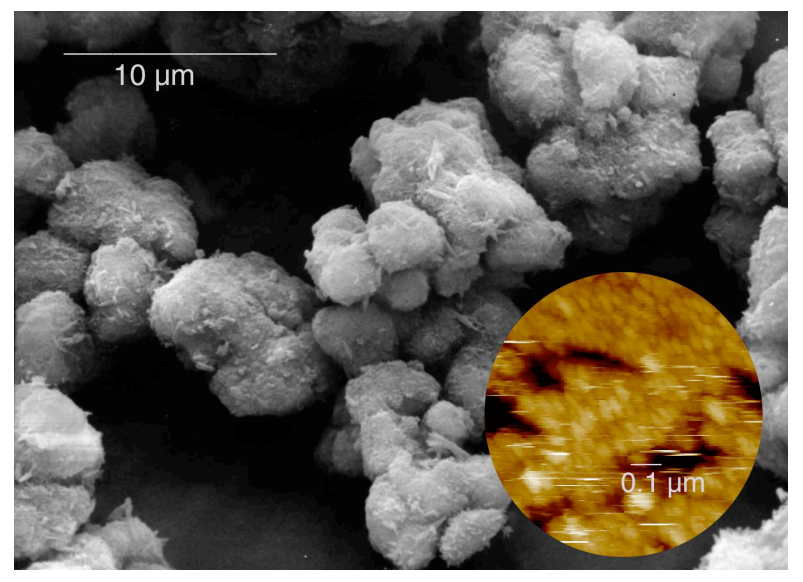

Table of Contents Graphics 